\title{
Variable Frequency Modulation for EMI Suppressing in Power Converter
}

\author{
Zhiwen Cao, Yiming Zhang \\ College of Electronic Information and Control Engineering, Beijing University of Technology, Beijing \\ Email: czw72413@163.com
}

Received February, 2013

\begin{abstract}
Switch mode power supply (SMPS) is good selection for power supplies of Unmanned Aerial Vehicle (UAV), which is one of the most important interference sources of UAV. The power switches with their high $\mathrm{dv} / \mathrm{dt}$ and di/dt switching slopes are the sources of electromagnetic interference (EMI). In this paper, a variable frequency modulation technology of the forward converter of UAV is presented, which is utilized in SMPS to improve electromagnetic compatibility (EMC). In variable-frequency techniques, power (signal) is transmitted in power converter in wide-band mode in several frequencies that are constantly changing, the EMI spectral performance of the SMPS can be controlled with the modulating pattern and modulation method. The validity of the models and analyses are confirmed experimentally by using a dc/dc forward converter.
\end{abstract}

Keywords: SMPS; Variable Frequency Modulation; EMI; EMC

\section{Introduction}

Recent advances made in semiconductor technology have led to substantial increases in the current and voltage ratings of gate controlled devices in Switch mode power supply (SMPS). Utility related applications of these types of devices have captured much attention from industry and academic communities. The reasons of the popularity of SMPS are efficiency, size, capability to operate at different currents and voltage levels, control features, and price compared to other possible solutions.

An Unmanned Aerial Vehicle (UAV) is a remotely operated vehicle or autonomous aircraft, and can re-use unmanned aircraft. UAV technology has been widely used for aerial reconnaissance, surveillance, communications, anti-submarine, electronic interference and other fields. Nowadays, the UAV has become one of the latest application technologies in the aeromagnetic field. The problem of magnetic interference of UAV must be solved to implement high-precision aeromagnetic surveys. The switch mode power supply (SMPS) is one of the most important interference sources of UAV [1].

UAV platforms usually dictate that all carried components should be light in weight, small in volume, and energy efficient. Because the inner space of a UAV is so confined, a smaller size of SMPS is required. The sizes of the energy storage elements (transformers, inductors, and capacitors) in a switch-mode power supply decrease in an approximately linear fashion along with the in- crease of the switching frequency. High-density power supplies of UAVs generally demand a high switching frequency and fast semiconductor devices.

DC-DC power converters are innate powerful sources of electromagnetic interferences because of the large $\mathrm{di} / \mathrm{dt}$ and $\mathrm{dv} / \mathrm{dt}$. Unlike linear regulators, which operate the power transistors in the linear mode, the Pulse Width Modulated (PWM) power supply operates the power transistors in both the saturated and cut-off states. SMPS are usually implemented with the use of constant clock frequency, so SMPS that have a periodic switching pattern, have an EMI spectrum that contains switching frequency and its harmonic frequencies. Electromagnetic Interference has become the major problem of switching mode power supplies in recent years. In order to reduce electromagnetic interference, Variable Frequency modulation is used in the paper, the EMI spectral performance of the SMPS can be effectively controlled. The control circuit of a pulse width modulated dc/dc converter is expected to be more complex than a control circuit used for the conventional PWM controlled dc/dc converter.

\section{Constant Pulse Width Modulation}

Advanced telecommunication and computer systems of UAV require high efficiency and power density, and distributed power supplies. Both the fly-back and forward converter topologies are good candidates for this application. The principal features of the supplies are a constant 
operating frequency, high efficiency and high power density. Forward switching power is supply from the buck converter, compared with fly back switching power supply, it has higher efficiency, the belt load capacity strongly and lower ripple factor, so it is the best choice.

\subsection{PWM Control Circuit}

As the $\mathrm{DC} / \mathrm{DC}$ converters follow the new trend of power generation, their switching frequencies have increased dramatically to reduce their dimensions. The increased switching frequency, together with the increased current and voltage slew rates, $\mathrm{di} / \mathrm{dt}$ and $\mathrm{dv} / \mathrm{dt}$, and consequently, wide bandwidths, have detrimental effects on the EMC performance of the power supplies. Electronic power converters are sources of electromagnetic interference at their input power source as well as in other neighboring electronic equipment, and suppression of EMI is a major issue in switch-mode power converter design.

The conventional PWM voltage control scheme is constant pulse width modulation. In this approach, the phase angle between the supply voltage and the current is maintained constant. The dc output voltage is controlled by varying the duty cycle of the conducting switches.

\subsection{PWM Control Circuit}

The inherent performance advantage of power MOSFETs makes their use very attractive in switched mode power supplies. The fundamental advantage of the power MOSFET is the operation at fast switching speeds and high frequencies. Higher operating frequency allows further reduction in the size of the filter and magnetic components. Figure 1 shows the circuit topology of a practical forward converter, which takes into account the non-ideal nature of a practical transformer. The control circuit is implemented by the use of the current-mode controller IC, UC3844 which is very popular in controlling power supplies. The performance of a power supply is dependent on various factors such as layout and transformer construction etc. It consists of a fast switching device ' $Q$ ' along with its control circuitry, and a transformer with its primary winding connected in series with switch ' $Q$ ' to the input supply.

The circuit uses an extra tertiary winding $\left(\mathrm{N}_{\mathrm{d}}\right)$ with a series diode to recover the energy when the switch is turned off. Usually, high voltage with twice the input DC voltage is used to obtain the maximum $50 \%$ duty ratio for resetting the transformer flux during the MOSFET's off period at low input DC voltage and full load. That means the peak voltage of twice the primary DC voltage is developed across the MOSFET during the off period. Mechanical switches (relays and circuit breakers) have a high inrush current (capacitive effect) at making contact and spark-over at the breaking contact (inductive effect), and cause wide-band emission with a continuous spectrum [4-5].

Switch mode power supplies are usually implemented with the use of constant clock frequency; for example, with traditional pulse width modulation (PWM). PWM signal is supplied from some special chip, for example, 3842. The modulator clock and harmonic frequencies are present in both conducted and radiated-EMI. SMPS that have a periodic switching pattern, have an EMI spectrum that contains switching frequency and its harmonic frequencies. These periodic noise components may be very harmful because they are repeating continuously - even if they have low amplitude and energy content.

\subsection{Frequency Spectrum Analysis of PWM Pulse}

The traditional pulse width modulation can be considered as a periodic trapezoidal pulse train. Trapezoidal current wave is shown in Figure 2, are defined as follows: $\mathrm{A}=$ amplitude, $t_{r}=$ rise time, $t_{d}=$ fall time, $\tau=$ width [9].

The rise time and fall time is not equal for the actual control signal. According to the actual situation, a laboratory prototype is constructed with the following specifications: the pulse frequency $\mathrm{f}=1 / \mathrm{T}=10 \mathrm{KHz}$, the turn-on time $\mathrm{t}_{\mathrm{on}}=\mathrm{t}_{\mathrm{r}}=10 \mathrm{~ns}$, the turn-off time $\mathrm{t}_{\mathrm{off}}=\mathrm{t}_{\mathrm{d}}=20 \mathrm{~ns}$, the amplitude of the current $i_{\mathrm{d}}=\mathrm{A}$. A suitable simulation program has been used to evaluate the variation of the spectrum according to the values of the pulse parameters. By means of the MATLAB program, we can derive the spectrum at each frequency point. We can see the actual PWM control signal contains the switching frequency and its harmonic components from the spectrum analysis diagram. The spectrum coefficients $C_{n}$ for such a waveform are:

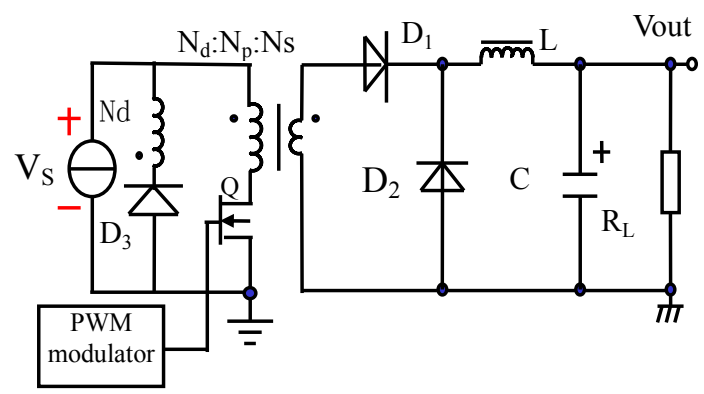

Figure 1. Circuit topology of a practical forward.

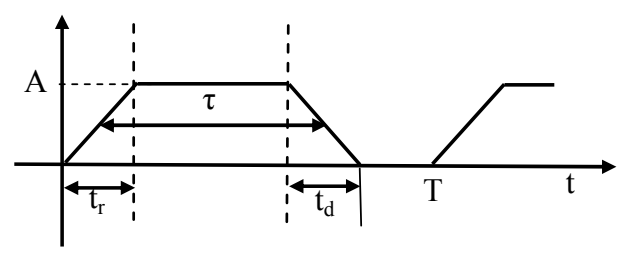

Figure 2. Trapezoidal pulse wave. 


$$
\begin{aligned}
& \mathbf{v}_{j_{\min }+1} C_{n}=-j \frac{1}{2 \pi n} e^{-j n \pi f_{0}\left(\tau+t_{o n}\right)} \\
& \cdot\left\{\left[\frac{d i_{d}}{d t}\right]_{o n} \frac{\sin \left(n \pi f_{0} t_{o n}\right)}{n \pi f_{0}} e^{j n \pi f_{0} \tau}-\left[\frac{d i_{d}}{d t}\right]_{o f f} \frac{\sin \left(n \pi f_{0} t_{o f f}\right)}{n \pi f_{0}} e^{-j n \pi f_{0} \tau}\right\} .
\end{aligned}
$$

Where:

$$
\left[\frac{d i_{d}}{d t}\right]_{\text {on }}=\frac{A}{t_{\text {on }}},\left[\frac{d i_{d}}{d t}\right]_{\text {off }}=\frac{A}{t_{\text {off }}}
$$

\section{Spread Spectrum Techniques}

From the discussion given in the last section, it is clear that the measured emission is periodical with respect to switching frequency. The emission, therefore, centers at the switching frequency and the harmonic frequencies. Concentration of emission power at these discrete frequencies makes it harder to meet EMI regulations.

\subsection{Frequency Spectrum Analysis of Spread Spectrum}

Usually, the SMPS are controlled by square switching signal with constant frequency and duty cycle (D) adjusted to the response of the control loop. One of the conventional techniques in order to reduce EMI consists of using passive filters, which has its limitations: size, weight, design complexity, efficiency, cost, etc. Modern variable-frequency (VF) EMI reduction techniques have been under intensive research to overcome the problems faced in filter-solutions. The interleaving technique is used to equally share the total power to be delivered the general idea in variable-frequency spread spectrum signaling is shown in Figure 3. Although the spectrum distribution of is different contained in Figures 3(a) and (b), but the total energy is the same in both cases, the peak level has reduced in Figure 3(b). The switching frequency modulation (SFM) is an effective method to reduce EMI in SMPS. This technique is based on the original spread-spectrum clock generation (SSCG) techniques. Using the SFM, there is a tradeoff between the amplitude reduction of the EMI harmonics and the generation of a set of additional side-band harmonics with small amplitude appears.

PWM spread spectrum control principle of SMPS can be described as below:

$$
f=f_{s}+\Delta f
$$

where $f_{s}$ is the reference frequency of the PWM switch; $\Delta f$ is the additional spread spectrum signal frequency, which is changing frequency according the time-domain characteristics of the spread spectrum signal. As is seen from the formula (3), The PWM spreading control is depending on the control of the $\Delta f$. The PWM control is the period spreading when $\Delta f$ is the period signal. The PWM control is the chaotic spreading when $\Delta f$ is the chaotic signal. As be shown in Figure 4, a pulsed process is constituted by spreading PWM, and can be expressed as the following pulse sequence:

$$
\xi(t)=\sum_{k=1}^{\infty} A \delta\left(t-\tau_{k-1}\right)
$$

Where in $\tau_{\mathrm{k}}$ represents the start time of the $\mathrm{k}$-th of the PWM waveform, A represents the amplitude of the drive pulse, $\mathrm{T}_{\mathrm{k}}$ is the $\mathrm{k}$-th pulse interval.

Corresponding to periodic or chaotic spread spectrum signal, the time interval $\mathrm{T}_{\mathrm{k}}$ of the PWM pulse respectively appear cycle changes or chaotic changes, the start time $\tau_{\mathrm{k}}$ of the $\mathrm{k}$-th drive waveform is the cumulative of the time intervals $T_{k}$, it is shown as below:

$$
\tau_{k}=\tau_{k+1}+\tau_{k-1}
$$

The period spreading can partly reduce the peak of the power spectrum of the PWM pulse, but it is still a discrete spectrum, the energy is concentrated in the $n f_{s} \pm k f_{m}$ specific frequency point, and did not get full extension, EMI can not meet the actual needs, so the article adopts chaotic control method. Chaotic control belongs to nonlinear control techniques in power electronics. These nonlinear control techniques generate non-harmonic switching spectrum when controller parameters are correctly chosen.

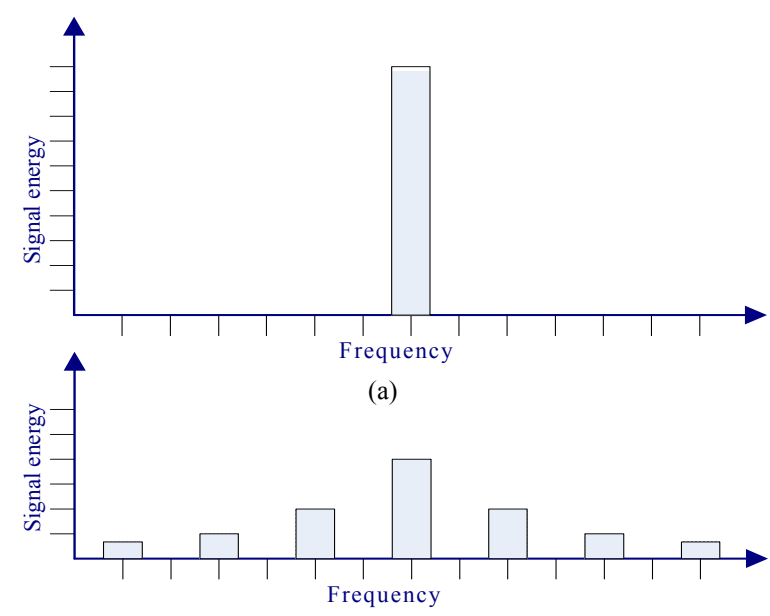

(b)

Figure 3. Spread spectrum signaling.

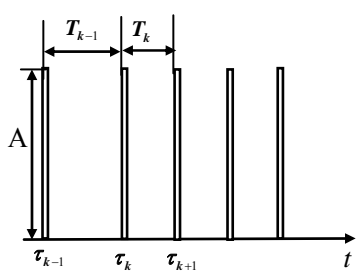

Figure 4. PWM drive pulses. 
The advantage of the non-linear control design approach is the simplicity of the circuit: even basic switch mode power supply control circuits, made by many IC manufacturers, can be used with only a few additional passive components. The main drawback of this approach is that the designer must study carefully the circuit performance at all load conditions and parameter variations to ensure the spread spectrum operation and system overall stability in all cases.

\subsection{Quantitative Analysis of Chaos Spread Spectrum}

As is shown in Figure 5, PWM pulse interval time $T_{k}$ under the control of the chaotic spread spectrum can be described as chaotic mapping $\varphi$ :

$$
T_{k+1}=\varphi\left(T_{k}\right)
$$

The K-th PWM drive pulse start time $\tau_{\mathrm{k}}$ is a chaotic sequence, as is shown in equation (7):

$$
\begin{aligned}
\tau_{k+1} & =T_{1}+\varphi\left(T_{1}\right)+\varphi^{(2)}\left(T_{1}\right)+\cdots+\varphi^{(k)}\left(T_{1}\right) \\
& =\varphi^{(k)}\left(T_{1}\right)
\end{aligned}
$$

Through analysis, we can get chaotic PWM pulse power spectral densities of formula:

$$
\begin{aligned}
S_{\xi}(\omega)= & \lim _{T \rightarrow \infty} \frac{1}{N \cdot E\left(T_{K}\right)} A^{2} E\left\{N+2 \sum_{L=1}^{\infty} P_{N(T)}(T)\right\} \\
& \times \sum_{i=1}^{N(T)-1} \sum_{i=1}^{N(T)-1} \cos \left(\omega\left(\tau_{i+K}-\tau_{i}\right)\right)
\end{aligned}
$$

where:

$$
\lim _{T \rightarrow \infty} \lim _{N(T) \rightarrow \infty} P_{N(T)}(T)=1, \quad N=E(N(T)), P_{N(T)}(T)
$$

represents the probability of the event

$$
\left\{\tau_{N(T)} \leq T \leq \tau_{N(T)+1}\right\},
$$

E represents the mathematical expectation here.

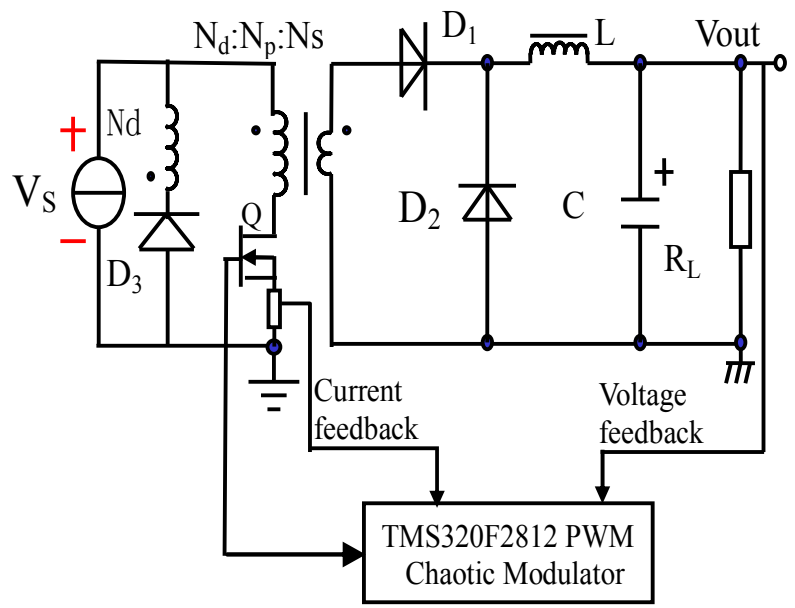

Figure 5. Schematic diagram of PWM Control System.
As can be seen from equation (7), chaotic spread spectrum control PWM pulse spectrum has the continuity characteristic, the power spectrum of chaotic spreading spectrum drive pulse is no longer concentrated in particular spectrum, but contains various frequency spectrum, the energy is no longer concentrated in specific frequency point, energy can be spread in the entire frequency range, so the chaotic spread spectrum is much better than period spread spectrum in EMI suppression. It became effective way to solve the EMI problem of switching converter.

\subsection{PWM Control System Structure}

Control schematic diagram based on TMS320F2812 is shown in Figure 1 in the forward converter. The TMS320 F2812 is a low-power 32-bit fixed-point digital signal processor. He focused on many of the outstanding features of the digital signal processing. The $\mathrm{C} 28 \mathrm{x}$ is a very efficient $\mathrm{C} / \mathrm{C}++$ engine, enabling users to develop not only their system control software in a high-level language, but also enables math algorithms to be developed using $\mathrm{C} / \mathrm{C}++$. The $\mathrm{C} 28 \mathrm{x}$ is as efficient in DSP math tasks as it is in system control tasks that typically are handled by microcontroller devices. Add to this the fast interrupt response with automatic context save of critical registers, resulting in a device that is capable of servicing many asynchronous events with minimal latency.

Chaos can be loosely defined as an apparently random behavior in a nonlinear system. Since all switch mode power electronic circuits are nonlinear, chaotic behavior can be expected in power electronic circuits with some specific component and parameter values. To achieve chaos control, the first work is to select the chaotic signals based on need to generate chaos equation. The chaos equation is very rich due to the long period development, for example: Lienard equations, Van Der Pol equations. The Lorenz equations is selected to produce chaotic signals in this paper, it is shown as below:

$$
\left\{\begin{array}{l}
\dot{x}=\sigma(y-x) \\
\dot{y}=\rho x-y-x z \\
\dot{z}=x y-\beta z
\end{array}\right.
$$

\subsection{Program}

The powerful computing power of DSP can be helpful to generate the chaotic signal, we usually adopt numerical calculation method. The program flow chart is shown in Figure 6, its core source program is shown as below: void main ( )

\{

int time $=1$;

double $\mathrm{x}=0.15$;

double $\mathrm{y}=0.1$; 
double $\mathrm{z}=0.1$;

int $\mathrm{i}$;

for $(i=1 ; i<10000000 ; i=i+$ timedelt $) \quad / /$

Iteration module \{

$\mathrm{x}=\mathrm{x}+$ time $* \quad(-16 * \mathrm{x}+16 * \mathrm{y}) / 1000$

$\mathrm{y}=\mathrm{y}+$ time $^{*} \quad(-1 * \mathrm{x} * \mathrm{z}+45.2 * \mathrm{x}-\mathrm{y}) / 1000$

$\mathrm{z}=\mathrm{z}+$ time $* \quad(\mathrm{x} * \mathrm{y}-4 * \mathrm{z}) / 1000$;

\}

\}

\subsection{Numerical Accuracy}

Chaotic signals were generated mainly by means of powerful DSP computing power, what kind of numerical methods can be used basing on the accuracy requirements. Usually, the higher the accuracy we demand, the greater is the amount of computation required; the frequency of the chaotic signals should be lower, so the appropriate accuracy can be selected according to the actual needs. The amount of computation can be directly determined by the speed of operation, namely determined by chaotic signal generation speed.

\section{Experimental Verification}

We can use a spectrum analyzer (4395A) to accept magnetic interference signals. The value of near-field radiated emissions can be detected by a Closed Field Probe (11945A) and a balanced loop antenna (11966B). As can be seen from Figure 7, the spectra plotted in Figure 14 show a strong reduction of magnetic radiated emission

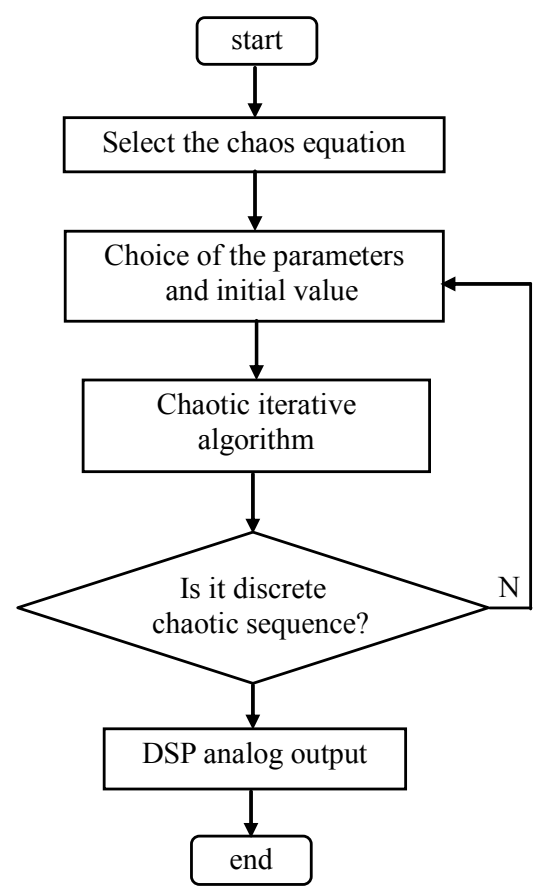

Figure 6. Program flow chart.

between $0.2 \mathrm{MHz}$ and $3 \mathrm{MHz}$. Noise energy is spread fur- there by chaotic spreading Spectrum, the noise of lower band noise wave widened, and the peak declined slightly; the spectrum peak of mid-frequency band dropped significantly.

\section{Conclusions}

This paper has given a detail to the effects of the chaotic control modulation schemes that are applied to $\mathrm{dc} / \mathrm{dc}$ converters operating in DCM. The chaotic control can fully spread the discrete frequency component in the classical PWM scheme to a continuous frequency spectrum. The chaotic control is a good choice because of its effectiveness in conducted EMI suppression and of its

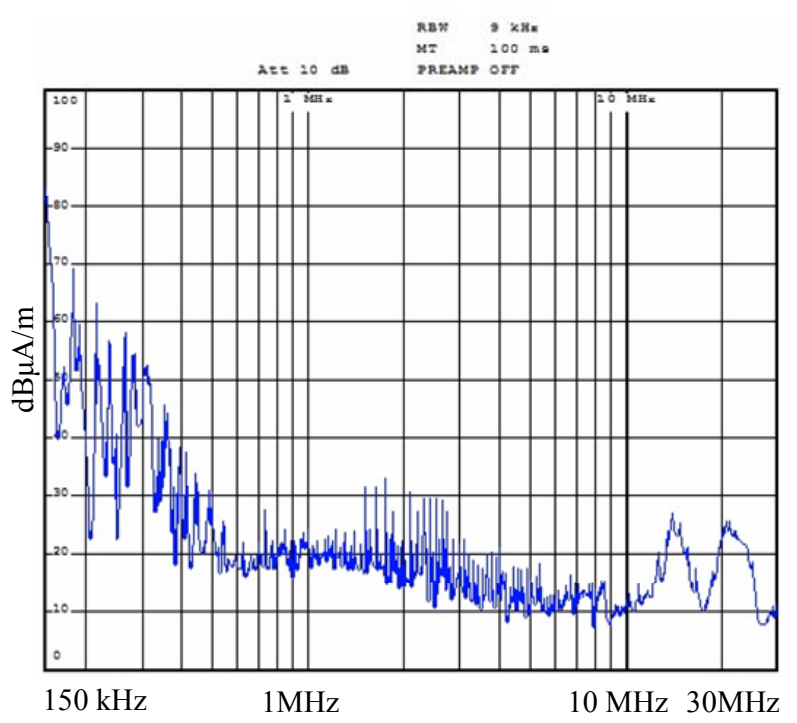

(a)

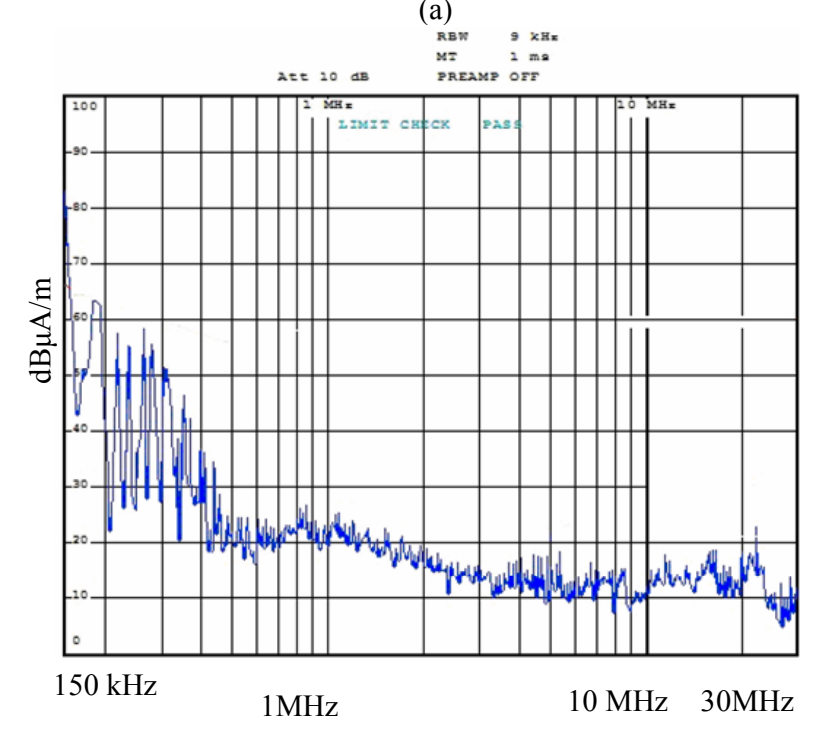

(b)

Figure 7. Near-field emissions measurement: (a) constant frequency (b) Chaos Spread Spectrum. ease in practical implementation. 


\section{Acknowledgements}

This article is supported jointly by SinoProbe-09-03 and Grant No. 201011080 (the development of the fixedwing UAV Aeromagnetic survey system). My deepest gratitude goes to Professor YiMing Zhang, my supervisor, for his constant encouragement and guidance. I also owe my sincere gratitude to my friends and my fellow classmates who gave me their help.

\section{REFERENCES}

[1] L. Beloqui and J. M. Usategui, "Vertical Differentiation and Entry Deterrence: Reconsideration," WP 2005-06, Dept. Fundamentos del Analisis Economico II, Universidad del Pais Vasco, 2005.

[2] R. Bergoeing and T. J. Kehoe, "Trade Theory and Trade Facts," Federal Reserve Bank of Minneapolis Research Department Staff Report, No. 284, 2003. 